\title{
Pyramidal Architectures for Computer Vision
}




\section{ADVANCES IN COMPUTER VISION AND MACHINE INTELLIGENCE}

Series Editor: Martin D. Levine

McGill University

Montreal, Quebec, Canada

\section{COMPUTER VISION FOR ELECTRONICS MANUFACTURING}

L. F. Pau

\section{HUMAN ENGINEERING IN STEREOSCOPIC VIEWING DEVICES}

Daniel B. Diner and Derek H. Fender

PYRAMIDAL ARCHITECTURES FOR COMPUTER VISION

Virginio Cantoni and Marco Ferretti

SIGMA: A Knowledge-Based Aerial Image Understanding System

Takashi Matsuyama and Vincent Shang-Shouq Hwang 


\title{
Pyramidal Architectures for
}

\author{
Computer Vision
}

\section{VIRGINIO CANTONI \\ and \\ MARCO FERRETTI}

University of Pavia

Pavia, Italy 


\section{Library of Congress Cataloging-in-Publication Data}

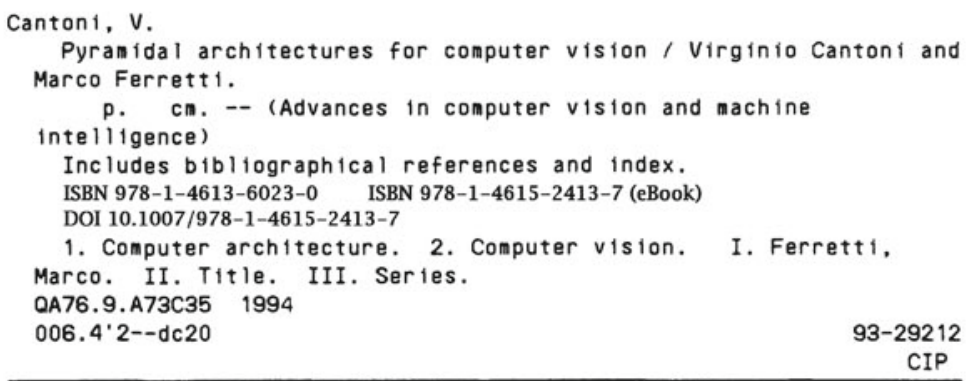

\section{All rights reserved}

No part of this book may be reproduced, stored in a retrieval system, or transmitted in any form or by any means, electronic, mechanical, photocopying, microfilming, recording, or otherwise, without written permission from the Publisher 
To Laura, Vera, and Livio -V.C.

To Elvira and Eleonora -M.F. 


\section{Preface}

Computer vision deals with the problem of manipulating information contained in large quantities of sensory data, where raw data emerge from the transducing sensors at rates between $10^{6}$ to $10^{7}$ pixels per second. Conventional generalpurpose computers are unable to achieve the computation rates required to operate in real time or even in near real time, so massively parallel systems have been used since their conception in this important practical application area.

The development of massively parallel computers was initially characterized by efforts to reach a speedup factor equal to the number of processing elements (linear scaling assumption). This behavior pattern can nearly be achieved only when there is a perfect match between the computational structure or data structure and the system architecture. The theory of hierarchical modular systems (HMSs) has shown that even a small number of hierarchical levels can sizably increase the effectiveness of very large systems. In fact, in the last decade several hierarchical architectures that support capabilities which can overcome performances gained with the assumption of linear scaling have been proposed. Of these architectures, the most commonly considered in computer vision is the one based on a very large number of processing elements (PEs) embedded in a pyramidal structure.

Pyramidal architectures supply the same image at different resolution levels, thus ensuring the use of the most appropriate resolution for the operation, task, and image at hand. Furthermore, a hierarchy is introduced by interplane communication which allows the implementation of a general "planning" strat- 
egy. Here the approach is to solve problems at a low spatial resolution, and therefore with a small amount of data, and then to proceed to successive refinements until the final verification of the results at the highest resolutions available. In this way, speedup factors greater than the number of PEs can be obtained in the quoted low-level vision tasks, because data reduction is an exponential function of the number of levels which have been used.

This approach has shown how a few hierarchical levels can substantially increase the processing efficiency of systems comprising many PEs; this behavior pattern has found a precise verification in various practical problems. Even in nature several large systems, composed of a great number of elements, have been shown to be self-organizing in a hierarchical structure, and the rules that these large sets configure follow the theory of HMSs.

This book is split logically into three sections, which consist of Chapters 1 and 2, Chapters 3 to 8 , and Chapters 9 and 10, respectively.

The first section provides the groundwork for the architectural analysis carried out in the remainder of the book. It discusses the role of the hierarchy in setting up complex systems and then specializes in the use of the hierarchy in computer vision systems.

Chapter 1 reviews the theory of hierarchical modular systems, which model the behavior of large self-organizing natural systems, such as monetary systems, settlement distribution over a territory, and natural languages. This theory shows that the introduction of a few hierarchical levels substantially increases the effectiveness of such large systems. The modularity criterion is inherited to some extent in the structure of most hierarchical architectures.

Chapter 2 discusses in depth the benefits of hierarchical strategies in the vision domain. Essentially, the motivation for using such an approach is that of obtaining high computational performances by processing only the relevant image data at the right time. The behavior of the known parts of the human vision system is used as a guideline. Its preattentive and attentive phases perform the basic tasks of any hierarchical processing paradigm - that is, delineating the region of interest and focusing on it for a detailed scrutiny. In computer vision, such a paradigm is supported by ad hoc data structures that consist of multiresolution grids, which reproduce the image in different amounts of details. The term pyramid is used to clarify the hierarchical connections between adjacent grid layers. This chapter analyzes the alternatives for building such representations and the possible processing strategies.

The second section more closely covers the architectural issues. It first introduces a framework for comparing the possible solutions according to topology and to its functional composition. Then it describes and discusses the 
actual machines and/or prototypes that can be described as pyramid architectures or that have a pyramid processing mode explicitly supported in hardware. The simulation of pyramids on other parallel systems is also covered in detail.

Chapter 3 describes all system organizations that construct a hierarchy with a homogeneous set of processing elements. In such cases, the topology of the interconnections is the main feature of the resulting system. The families of hierarchical systems considered include snowflakes, stars, trees, hypernets, and pyramids. A quantitative assessment of these hierarchies is carried out through a set of parameters that measure the capability of the network to sustain data exchanges among the processing elements.

Chapter 4 focuses on hierarchical machines that have already been built (or at least fully designed) and organizes them into a taxonomy. This taxonomy is itself a small hierarchy with two levels. The first level splits the systems into homogeneous or heterogeneous ones according to their processing module capability. The second is based on the means of coupling these modules and on interconnection networks (tight-loose, compact-distributed, fixed-reconfigurable). The processing paradigm varies within the taxonomy: these include pipeline, SIMD, multi-SIMD, and MIMD systems.

Chapter 5 concentrates on the most popular hierarchical topology, the basic pyramid, and on the homogeneous, massively parallel systems that have been proposed or at least built in prototype. When designing a pyramid structure, one may follow two approaches: the first with fine granularity, where one processor per image pixel is conceived, and the second with coarse granularity, where one microprocessor is associated with an image block. The former approach is the one followed in most cases, perhaps because of the expected benefits in designing a parallel system with VLSI. This chapter offers a comprehensive and in-depth analysis of the actual pyramid computers that attracted so much interest in the mid- and late-1980s.

Chapter 6 analyzes alternatives to the true pyramid computer. Specialized hardware solutions have been proposed to achieve multiresolution processing without resorting to these expensive parallel architectures. Pipelined systems specializing in decimation (pyramid building) and expansion are the most effective alternative. The processing facilities of such systems offer a powerful multiresolution environment that can be easily integrated into low-cost, application-specific devices.

Chapter 7 addresses a more pragmatic issue. Massively parallel systems, once a small niche in the computer processing community, have now come of age, and commercial systems have become more and more widespread. Since none of them adopts a pyramid topology, it is worth studying the cost of em- 
bedding pyramids into their native structure. The mesh and the hypercube are the two most common topologies of such commercial systems. This chapter reviews embeddings proposed for the basic pyramids in these topologies.

Chapter 8 closes the central section of the book by analyzing heterogeneous hierarchical systems. The rationale for designing a hierarchical system according to this paradigm is that it is difficult to match the computational requirements of the various processing steps (low to high) in a vision problem with just a single homogeneous architecture. Low-level tasks demand specialized hardware capable of matching the high speed of incoming data. Subsequent processes are best matched onto coarse-grained standard microprocessors. Some systems have been designed to merge the pyramid concept with this heterogeneous structure.

The third section covers the user's point of view. Programming tools, including languages and development tools, are notoriously the most difficult part of a system project. With the recent advances in VLSI design, the time required to specify, design, build, and assemble a functioning prototype is shorter by order of magnitudes than the corresponding time to obtain a standard compiler. However, effective use of the hierarchical system must be made possible to the "naive" end user, whose energy should only be concentrated on the tasks, with almost no regard to the intricacies of the complex system he or she is programming.

Chapter 9 covers the language side of this problem. Specific attempts have been made to conjugate standard languages with the pyramid architecture. Both the data structures and the semantics of the control operators need a revised interpretation.

Chapter 10 faces the ultimate question of multiresolution processing. The expected advantages of this processing strategy must be measured (both from a theoretical computational point of view and in practical situations) and proved to be relevant. The chapter contains a selection from the huge number of algorithms that exploit pyramid processing in very diverse computer vision contexts. This overview, which does not pretend to be an exhaustive and up-todate tutorial on the subject, focuses on those algorithms which apply the multiprocessing strategy at its best.

Virginio Cantoni

Marco Ferretti

Pavia, Italy 


\section{Acknowledgments}

Many are the people who contributed to the effort of setting up this book. Some kindly provided up-to-date material on their work; others simply helped informally with suggestions. Among them, we would like to thank particularly Dr. Angelo Buizza for his guidance through the biological aspects of human vision, Dr. Mauro Mosconi, and our co-workers in the Computer Vision and CAD Laboratories of Pavia University. 


\section{Contents}

1. Hierarchical Architectures. . . . . . . . . . . . . . 1

1.1. Introduction . . . . . . . . . . . . . 1

1.2. Theory of Hierarchical Modular Systems . . . . . . . . . . 3

1.3. Self-Organizing Hierarchical Modular Systems . . . . . . . . 6

1.3.1. The Invariance Principle and the Law of Distribution. . . 6

1.3.2. Some Examples of HMSs in Society. . . . . . . . . . 8

1.4. Hierarchical Architectures for Parallel Processing Machines . . . 10

References . . . . . . . . . . . . . . 11

2. Hierarchical Strategies in Computer Vision Systems. . . . . . . 13

2.1. Introduction . . . . . . . . . . . . . . 13

2.1.1. Recognition Cones . . . . . . . . . . . . . . . 14

2.1.2. Alerting Mechanisms and Peripheral Guidance . . . . . 18

2.2. Allocation of Attention in Computer Vision Systems . . . . . 24

2.2.1. Multiresolution Model Representations. . . . . . . . . . 26

2.3. Multiresolution Matching. . . . . . . . . . . . . . . 33

2.4. Fine-to-Coarse Feature Generation . . . . . . . . . . . . 34

2.4.1. Wavelet Representation . . . . . . . . . . . 35

2.4.2. Gaussian Pyramid. . . . . . . . . . . . . . 41

2.4.3. Laplacian Pyramid . . . . . . . . . . . . . . . . . . . . . . . . . . . . .

2.4.4. Haar Pyramid. . . . . . . . . . . . . . . . 51 
2.4.5. Feature Pyramid . . . . . . . . . . . . . 56

2.5. Coarse-to-Fine Searches . . . . . . . . . . . . . . 57

2.6. Image Flow Diagrams . . . . . . . . . . . . . . 60

2.6.1. Examples . . . . . . . . . . . . . . 60

2.7. General Planning Strategies . . . . . . . . . . . . 63

References . . . . . . . . . . . . . . 65

3. Hierarchical Homogeneous Topologies. . . . . . . . . . . 69

3.1. Introduction . . . . . . . . . . . . . . 69

3.2. Hierarchical Paradigm . . . . . . . . . . . . . . . 70

3.3. Comparison Parameters and Evaluation Criteria . . . . . . . 71

3.4. Bus-Oriented Architectures. . . . . . . . . . . . . . . 74

3.4.1. Snowflakes and Dense Snowflakes. . . . . . . . . . 74

3.4.2. Partial and Full Stars . . . . . . . . . . . . . . . 77

3.5. Link-Oriented Architectures . . . . . . . . . . . . . . 79

3.5.1. Regular Trees. . . . . . . . . . . . . . . . 79

3.5.2. Augmented Trees . . . . . . . . . . . . . . 81

3.5.3. Complete Trees. . . . . . . . . . . . . . 84

3.5.4. Pyramids . . . . . . . . . . . . 86

3.5.5. Hypernets . . . . . . . . . . . . . . . . 89 89

3.6. Performance Measures . . . . . . . . . . . . . . . 92

3.7. Applicability . . . . . . . . . . . . . . . . 997

3.7.1. VLSI Feasibility . . . . . . . . . . . . . . 97

3.7.2. Applications . . . . . . . . . . . . . . 98

3.7.3. Prototypes and Machines . . . . . . . . . . . . . . . 99

3.8. Conclusions . . . . . . . . . . . . . . . . 100

References ........................ 100

4. A Taxonomy of Hierarchical Machines for Computer Vision . . . 103

4.1. Paradigm for Computer Vision . . . . . . . . . . . . . . . . . . . . . . . .

4.1.1. Preprocessing . . . . . . . . . . . . . . . 104

4.1.2. Intermediate-Level Processing . . . . . . . . . . . . 104

4.1.3. High-Level Processing . . . . . . . . . . . . . . . 105

4.1.4. Three Possible Computational Frameworks. . . . . . . . 106

4.2. Taxonomy of Hierarchical Machines . . . . . . . . . . . . 106

4.2.1. Heterogeneous Loosely Coupled Class. . . . . . . . . . 109

4.2.2. Heterogeneous Closely Coupled Class . . . . . . . . . 110 
4.2.3. Homogeneous Compact Pyramid . . . . . . . . . . . . 110

4.2.4. Homogeneous Distributed Pyramid . . . . . . . . . . . 112

4.3. Conclusions . . . . . . . . . . . . . . . . . . 113

References . . . . . . . . . . . . . . . . . 114

5. Compact and Distributed Pyramids . . . . . . . . . . . . 117

5.1. Introduction . . . . . . . . . . . . . . 117

5.2. Compact Pyramids. . . . . . . . . . . . . . . .118

5.2.1. Interconnection Topology . . . . . . . . . . . . . . 119

5.2.2. Processing Element Capabilities . . . . . . . . . . . 120

5.2.3. System Configuration . . . . . . . . . . . . . . . . 125

5.2.4. Compact Pyramid Prototypes . . . . . . . . . . . . 129

5.3. Distributed Pyramids. . . . . . . . . . . . . . . 154

5.3.1. The EGPA System . . . . . . . . . . . . . . . . . . . . . . . . . .

5.4. Conclusions . . . . . . . . . . . . . . . . 158

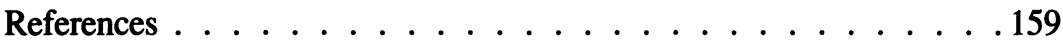

6. Pipeline Multiresolution Systems. . . . . . . . . . . . . . . 161

6.1. Introduction . . . . . . . . . . . . . . . 161

6.2. Pyramid Vision Machine System . . . . . . . . . . . . . 163

6.2.1. The Segmented Pipeline Architecture . . . . . . . . 165

6.2.2. The CAIP System. . . . . . . . . . . . . . . . 167

6.3. PIPE System . . . . . . . . . . . . . . . . . 168

6.3.1. The PIPE Architecture . . . . . . . . . . . . . . . 168

6.3.2. Pyramid Neighbor Operations . . . . . . . . . . . . . 170

6.4. Conclusions . . . . . . . . . . . . . . 171

References . . . . . . . . . . . . . . . . 171

7. Simulation of Pyramids on Flat Arrays and Hypercubes. . . . . . 173

7.1. Introduction . . . . . . . . . . . . . . . . 173

7.2. Pyramids and Meshes . . . . . . . . . . . . . . 175

7.2.1. Simulation of Pyramids on Flat Arrays. . . . . . . . . 175

7.2.2. Augmented Flat Arrays . . . . . . . . . . . . . . 181

7.2.3. Reconfigurable Meshes Emulating Pyramids . . . . . . . 183

7.3. Pyramids and Hypercubes . . . . . . . . . . . . . . . 192

7.3.1. Mesh-Based Embeddings . . . . . . . . . . . . . 194 
7.3.2. Other Embeddings . . . . . . . . . . . . . . . 199

7.3.3. Embeddings on Real Systems . . . . . . . . . . . . . 203

7.3.4. The Neighbor Addressing Scheme. . . . . . . . . . 215

7.4. Conclusions . . . . . . . . . . . . . . . . . . 216

References . . . . . . . . . . . . . . 216

8. Heterogeneous Hierarchical Systems. . . . . . . . . . . . . 219

8.1. Introduction . . . . . . . . . . . . . . . . 219

8.2. Warwick Pyramid System . . . . . . . . . . . . . 221

8.2.1. Structure of a Cluster . . . . . . . . . . . . 222

8.2.2. Clusters Interconnection. . . . . . . . . . . . . . 224

8.2.3. Programming Environment . . . . . . . . . . . 225

8.3. Image Understanding Architecture . . . . . . . . . . . 226

8.3.1. Three-Level Hierarchy . . . . . . . . . . . . . . .226

8.3.2. Array Control Unit and Programming Environment. . . . 231

8.4. The PASM . . . . . . . . . . . . . . . . . . 232

8.4.1. Partitionable Two-Level Hierarchy. . . . . . . . . . . 233

8.5. Array/Net Project . . . . . . . . . . . . . . . . . . 236

8.6. Conclusions . . . . . . . . . . . . . . . 238

References . . . . . . . . . . . . . . . 238

9. Programming a Hierarchical Structure . . . . . . . . . . . .241

9.1. Languages: An Introduction . . . . . . . . . . . . . . 241

9.1.1. Collection-Oriented Languages versus

Processor-Oriented Languages. . . . . . . . . . . . 242

9.1.2. Semantics of Parallel Constructs. . . . . . . . . . . 245

9.1.3. HCL, a Pyramid Algebra . . . . . . . . . . . . . . 250

9.1.4. HCL and the C Language . . . . . . . . . . . . . . 257

9.1.5. PYR-E . . . . . . . . . . . . . . 263

9.1.6. PCL . . . . . . . . . . . . . . . . 273

9.2. Control Environment. . . . . . . . . . . . . . . . . . . 277

9.2.1. Control of Multi-SIMD Hierarchical Systems . . . . . . 278

9.2.2. Multi-SIMD Control across Resolution. . . . . . . . . . 279

9.2.3. Multi-SIMD Control across Space . . . . . . . . . . . 286

9.3. Conclusions . . . . . . . . . . . . . . . . . . . 287

References . . . . . . . . . . . . . . . . 288 
10. Pyramidal Tools and Applications . . . . . . . . . . . . . 291

10.1. Introduction . . . . . . . . . . . . . . . . 291

10.2. Complexity of Some Basic Algorithms . . . . . . . . . . 292

10.3. Special Pyramids . . . . . . . . . . . . . . . . . . . . 294

10.3.1. Overlapped and Dual Pyramids . . . . . . . . . . . . 294

10.3.2. Stochastic, Adaptive, and Custom-Made Pyramids . . . 295

10.3.3. Centralization Graphs. . . . . . . . . . . . . . . . . . . . 297

10.4. Pyramidal Techniques. . . . . . . . . . . . . . . . . . 299

10.4.1. Multigrid Numerical Methods. . . . . . . . . . . . . 299

10.4.2. Image Segmentation . . . . . . . . . . . . . . . 301

10.4.3. General Matching . . . . . . . . . . . . . . . 308

10.4.4. Lines, Curves, and Shapes:

Description and Recognition . . . . . . . . . . . 309

10.4.5. Image Compression, Coding, and Transmission . . . . 315

10.4.6. Motion Analysis . . . . . . . . . . . . . 316

10.4.7. Stereo Vision and Depth . . . . . . . . . . 324

10.5. Conclusions . . . . . . . . . . . . . . 326

References . . . . . . . . . . . . . . . . 327

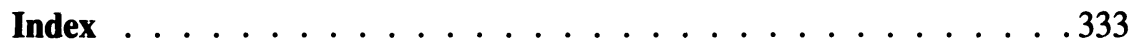




\section{Pyramidal Architectures for Computer Vision}

